\title{
Ageing assessment of zirconia implant prostheses by three different quantitative assessment techniques
}

\author{
Phyu Phyu Kyaw', Pong Pongprueksa', Warangkana Anuchitolarn², Krongkarn Sirinukunwatta ${ }^{3}$, \\ Kallaya Suputtamongkol ${ }^{1 *}$ \\ ${ }^{1}$ Faculty of Dentistry, Mahidol University, Rachathevi, Bangkok, Thailand \\ ${ }^{2}$ Chemical and Biological Analysis Section, Technical Support for Material Analysis Division, National Metal and Materials \\ Technology Center, Pathum Thani, Thailand \\ ${ }^{3}$ Department of Science Service, Ministry of Science and Technology, Ratchathewi, Bangkok, Thailand
}

\begin{abstract}
PURPOSE. To evaluate the influence of cyclic loading on phase transformation of zirconia abutments and to compare the effectiveness of three different quantitative ageing assessment techniques. MATERIALS AND METHODS. Thirty two Y-TZP prostheses fabricated from two brands, InCoris ZI and Ceramill Zl, were cemented to titanium bases and equally divided into two subgroups $(n=8)$ : control group without any treatment and aged group with cyclic loading between $20 \mathrm{~N}$ and $98 \mathrm{~N}$ for 100,000 cycles at $4 \mathrm{~Hz}$ in distilled water at $37^{\circ} \mathrm{C}$. The tetragonal-to-monoclinic phase transformation was assessed by (i) conventional x-ray diffraction (XRD), (ii) micro $\mathrm{X}$-ray diffraction $(\mu \mathrm{XRD})$, and (iii) micro-Raman spectroscopy. The monoclinic-phase fractions (M\%) were compared by two-way ANOVA. RESULTS. InCoris Zi presented significantly higher M\% than Ceramill Zi in both control and aged groups $(P<.001)$. Both materials exhibited significant phase transformation with monoclinicphase of 1 to $3 \%$ more in aged groups than controls for all three assessment techniques. The comparable M\% was quantified by both $\mu$ XRD and XRD. The highest M\% was assessed with micro-Raman. CONCLUSION. Cyclic loading produced significant phase transformation in tested Y-TZP prostheses. The micro-Raman spectroscopy could be used as an alternative to XRD and $\mu$ XRD. [J Adv Prosthodont 2019;11:253-61]
\end{abstract}

KEYWORDS: Y-TZP ceramics; Cyclic loading; Phase transformation

\section{INTRODUCTION}

As an implant abutment material, titanium is a traditional material of choice due to its excellent mechanical properties. ${ }^{1}$ Titanium abutments are also favorable for the periimplant soft tissues health and biocompatibility by deterring the incidence of galvanic and corrosive reactions in the

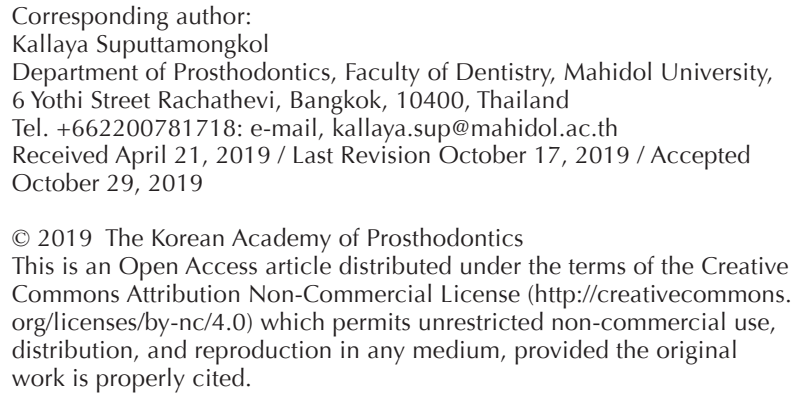

implant/abutment interface. Yet, there may be a problem in the titanium/porcelain systems because of excessive oxidation of this material at ceramic melting temperatures and the low adhesion of the oxides to the surface of it. ${ }^{2}$ In addition, metal abutments could not fully satisfy the esthetical, functional, and hygienic requirement fundamental to the implant-restorations success. To provide metal-free restorations with more esthetically pleasing results, tooth-colored implant abutments and prostheses have become fascinating alternatives. ${ }^{3}$ Amongst high strength ceramics, zirconia is particularly favorable due to its high strength and high fracture toughness. ${ }^{4}$

However, an occurrence of ageing or low temperature degradation (LTD) of yttria-stabilized tetragonal zirconia polycrystals (Y-TZP) due to a slow tetragonal to monoclinic $(\mathrm{t}-\mathrm{m})$ phase transformation in a humid environment at relatively low temperatures $\left(150-400^{\circ} \mathrm{C}\right)$ has drawn an enormous attention from the researchers. ${ }^{5,6,7}$ The concern about the lifetime of zirconia-based prostheses has been raised in 
medical and dental fields after distribution of the reports on premature failure of zirconia hip implants ${ }^{8}$ and FDA announcement for zirconia femoral heads fracture due to LTD. ${ }^{9}$ LTD of zirconia typically initiates at the surface of polycrystalline zirconia and then progresses toward the bulk of the material. The transformation of one grain leads to an increase in volume that causes stresses on the nearby grains and microcracking. This offers a path for the water to penetrate and then exacerbates the process of surface degradation and the transformation progresses from neighbor to neighbor. ${ }^{5,7}$ Studies have shown that an increase in monoclinic content is directly related to the degradation of zirconia material. ${ }^{10-14}$

In dentistry, clinical studies following the use of zirconia frameworks for posterior fixed partial dentures for more than 5 years $^{15}$ and Y-TZP abutments supporting single tooth crowns for 11 years ${ }^{16}$ have revealed the excellent clinical success of zirconia prostheses. With an increased interest of zirconia to be used as an implant prosthesis, researches have paid much attention on LTD and the effect of longterm aging on this material. Although some studies have stated autoclaving as an effective ageing protocol, ${ }^{7,10-14}$ the ageing results that measured well above the room temperature or body temperature could not reflect in vivo oral conditions. For better understanding of ageing process of dental zirconia, in vitro study's the ageing conditions should resemble the oral environments. For this reason, zirconia implant prostheses should be tested under cyclic loading in the presence of moisture at human body temperature in order to simulate oral environments.

The objectives of this study were to study the effects of cyclic loading on zirconia implant prostheses fabricated by two different computer aided-design and computer-aided manufacturing systems (CAD/CAM) and to assess the effectiveness of three different ageing assessment techniques for quantitative phase transformation analysis. To fulfill these objectives, different ageing assessment techniques - conventional X-ray diffraction, micro X-ray diffrac- tion, Raman microscopy and scanning electron microscopy - were used in this study.

\section{MATERIAL AND METHODS}

An implant replica for fixture diameter of $3.5 \mathrm{~mm}$ (Astra Tech, Dentsply, Bensheim, Germany) was embedded in polyvinyl chloride cylinder using autopolymerized epoxy resin. Each replica was embedded $3 \mathrm{~mm}$ below the implant shoulder in order to simulate bone resorption according to the ISO-Norm 14801. ${ }^{17}$ The replica was then assembled with a titanium base (AT OS 3.5/4.0 L, Sirona, Bensheim, Germany) (Fig. 1A) to be scanned and designed for fabrication of implant-supported zirconia prostheses from two different brands of presintered yttria-stabilized zirconia blocks (inCoris ZI meso, Sirona, Bensheim, Germany and Ceramill Zi, Amann Girrbach, Koblach, Australia) using Sirona and Amann Girrbach CAD/CAM systems respectively. The material composition and density is shown in Table 1.The prosthesis design is shown in Fig. 1B with 30-degree angulation at the occlusal surface. After designing and milling of pre-sintered zirconia blocks, the zirconia prostheses were sintered to obtain the final density and dimension according to a manufacturer's instruction. Then, the prostheses fabricated from each CAD/CAM system were sandblasted at the gluing surfaces with $50 \mu \mathrm{m}$ aluminum oxide at $2.0 \mathrm{bar}$, cemented to the titanium bases using a resin cement (Panavia F2.0, Kuraray, Osaka, Japan), and equally divided into two subgroups $(n=8)$ : the control with no cyclic loading group and the mechanical ageing group. For the mechanical ageing, the cyclic loading was applied on the zirconia prostheses with a tungsten carbide ball $(4.75 \mathrm{~mm}$ in diameter) for 100,000 cycles with the load between 20 and $98 \mathrm{~N}$ at $4 \mathrm{~Hz}$ in distilled water at $37^{\circ} \mathrm{C}$ using a servo hydraulic testing machine (Model E10000, Instron Corp., London, $\mathrm{UK})$. These prostheses were loaded at lingual surfaces with a $30^{\circ}$ angulation to the long axis as shown in Fig. $1 \mathrm{C}$.
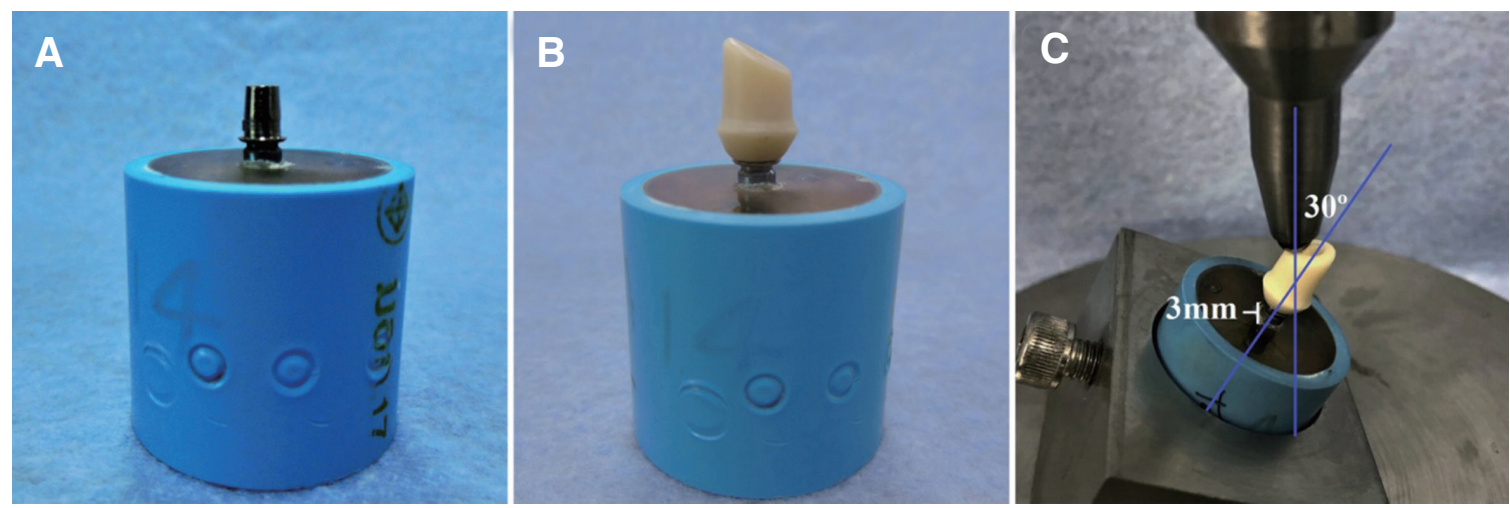

Fig. 1. An implant replica with a titanium base embedded in polyvinyl chloride cylinder (A) without a zirconia abutment (B) with a zirconia abutment and (C) a set up for cyclic loading. 
Phase transformation was assessed on the flat area at the lingual aspect of each prosthesis using $\mathrm{X}$-ray diffraction technique with $\mathrm{CuK} \alpha 1.5418 \AA$, at $40 \mathrm{kV}$, and $40 \mathrm{~mA}$ (Bruker AXS, D75181, Karlsruhe, Germany). The diffraction data were collected by two techniques: (i) conventional $\mathrm{X}$-ray diffraction (XRD) and (ii) micro $\mathrm{X}$-ray diffraction ( $\mu \mathrm{XRD})$.

For XRD, the diffraction data were collected conventionally in the $2 \theta$ range of 20 to 80 degrees, with a step size of 0.01 degrees and a counting time of $1 \mathrm{~s} / \mathrm{step}$. For $\mu$ XRD, the Gobel mirror parallel beam optics with exchangeable pinhole collimator of $100 \mu \mathrm{m}$ inner diameter and two dimensional general area detector diffraction system (GADDS) were used. Data were collected with a fixed incident angle of $15^{\circ}$ to the flat lingual surface (at the cyclic loading area). $\mu \mathrm{XRD}$ profiles were generated from 20 to 80 degrees with a step size of 0.01 degrees and a scan speed of $1 \mathrm{~s} / \mathrm{step}$.

Monoclinic phase fraction was determined from both $\mathrm{XRD}$ and $\mu \mathrm{XRD}$ data using the Match phase identification software version 3.3 (Crystal Impact, Bonn, Germany) with the standard models recorded in the Crystallography Open Database (COD) and Rietveld refinement method. The refined parameters were scale factors, specimen displacement, unit cell parameters, background, profile shaped, overall isotropic displacement, and preferred orientation.

The Raman Spectrophotometer (Senterra, BrukerOptik, Ettlingen, Germany) was used to perform a phase analysis using an argon laser with a wavelength of $532 \mathrm{~nm}$ and 20 $\mathrm{mW}$ power. An optical microscope with $100 \times$ objective was used to focus the laser spot on the prosthesis surface (at the cyclic loading area). The specimen integration time was $40 \mathrm{~s}$. The spectra were collected from 3 consecutive spots on the cyclically loaded surface of each specimen with a pinhole aperture of $50 \mu \mathrm{m}$. The monoclinic phase concentration $\left(C_{m}\right)$ was calculated according to Equation $1 .^{18}$

$$
C_{m}=\frac{I_{m}^{181}+I_{m}^{192}}{0.32\left(I_{t}^{148}+I_{t}^{264}\right)+I_{m}^{181}+I_{m}^{192}}
$$

where $I_{m}$ and $I_{t}$ represent the integrated intensities of the monoclinic and tetragonal peaks, respectively. The superscripts refer to the Raman shift of the characteristic peaks. In order to gain more information on the monoclinic phase transformation, the micro-Raman spectral mapping at the cyclically loaded area (approximately $100 \mu \mathrm{m}^{2}$ ) of a zirconia prosthesis in each experimental group was performed with a Raman spectrometer (LabRAM HR Evolution, Horiba Instruments Pte Ltd., Singapore). An area of interest was selected and images (20 points per image) were recorded with a $785 \mathrm{~nm}$-wavelength laser with an acquisition time of 30 - 60 s using a $50 \times$ objective.

The representative specimens from each group were prepared for a surface evaluation using the scanning electron microscopy (SEM). They were cleaned in an ultrasonic cleanser with absolute ethanol for $10 \mathrm{~min}$ and then air-blow dried. Then the zirconia prostheses were fixed on a metal stub with carbon adhesive tape and coated with gold in a sputter coater (PolaronSC7620, Quorum Technologies Ltd., East Sussex, UK), and observed using a scanning electron microscope (JSM-6610LV, JEOL USA Co. Ltd., Peabody, MA, USA) under high vacuum and a secondary electron imaging mode with an accelerating voltage of $20 \mathrm{kV}$.

Monoclinic phase fractions of all experimental groups were analyzed by the Shapiro-Wilk normality tests and twoway ANOVA to determine the difference with regard to material and cyclic loading within each ageing assessment technique at $\alpha=0.05$.

\section{RESULTS}

The tetragonal and monoclinic peaks were detected in all specimens of both control and cyclically loaded groups assessed by two x-ray diffraction techniques as shown in Fig. 2 and Fig. 3. The monoclinic phase fractions observed in each group are summarized in Table 2.

For the Raman spectroscopy, the typical Raman bands of tetragonal phase were clearly observed for all specimens. The monoclinic doublets were evident in cyclically loaded specimens as shown in Fig. 4. However, the monoclinic phase intensity of the same specimen collected from the three consecutive points in the cyclic-loading area was varied and the variation is illustrated in Fig. 5. In this case, the highest monoclinic phase intensity was used to represent the amount of phase transformation from a cyclic loading. The monoclinic phase contents calculated according to Equation 1 of all groups are summarized in Table 2. The micro-Raman spectral images are shown in Fig. 6. The patterns of monoclinic phase distribtion appeared to be concentrated at the protruded surface (pointed by the arrows) of both InCoris ZI and Ceramill ZI.

The results obtained from two-way ANOVA indicated that the material and cyclic loading had significant effects on the amount of monoclinic phase transformation obtained from all ageing assessment techniques except those obtained from the micro-Raman technique $(P<.03)$. InCoris ZI exhibited significantly greater amounts of monoclinic phase than Ceramill ZI for both control and cyclically loaded groups. After cyclic loading, a significant increase in monoclinic phase was also observed for both InCoris ZI

Table 1. Normal composition and density of Y-TZP materials stated by manufacturers

\begin{tabular}{ccc}
\hline Composition & Ceramill $\mathrm{Zl}$ & InCoris $\mathrm{Zl}$ \\
\hline $\mathrm{ZrO}_{2}+\mathrm{HfO}_{2}+\mathrm{Y}_{2} \mathrm{O}_{3}$ & $>99 \%$ & $\geq 99.0 \%$ \\
$\mathrm{Y}_{2} \mathrm{O}_{3}$ & $4.5-5.6 \%$ & $4.5-6 \%$ \\
$\mathrm{HfO}_{2}$ & $<5 \%$ & $\leq 5 \%$ \\
$\mathrm{Al}_{2} \mathrm{O}_{3}$ & $<0.5 \%$ & $\leq 0.5 \%$ \\
Other oxides & $<0.5 \%$ & $\leq 0.3 \%$ \\
Density & $6.05 \mathrm{gcm}^{-3}$ & $6.05 \pm 0.2 \mathrm{gcm}^{-3}$ \\
\hline
\end{tabular}



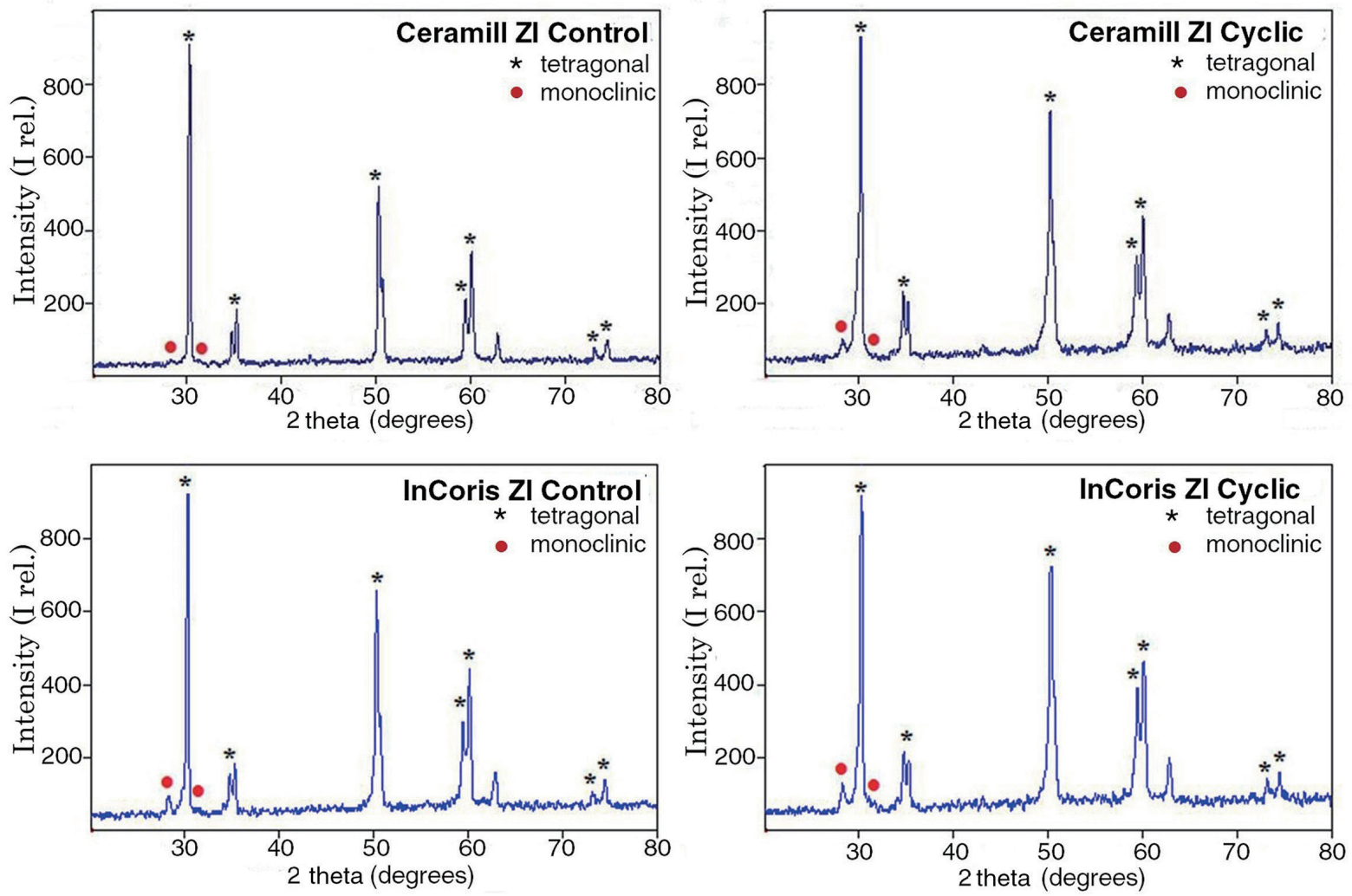

Fig. 2. The representative XRD patterns obtained from the surface of zirconia specimens.
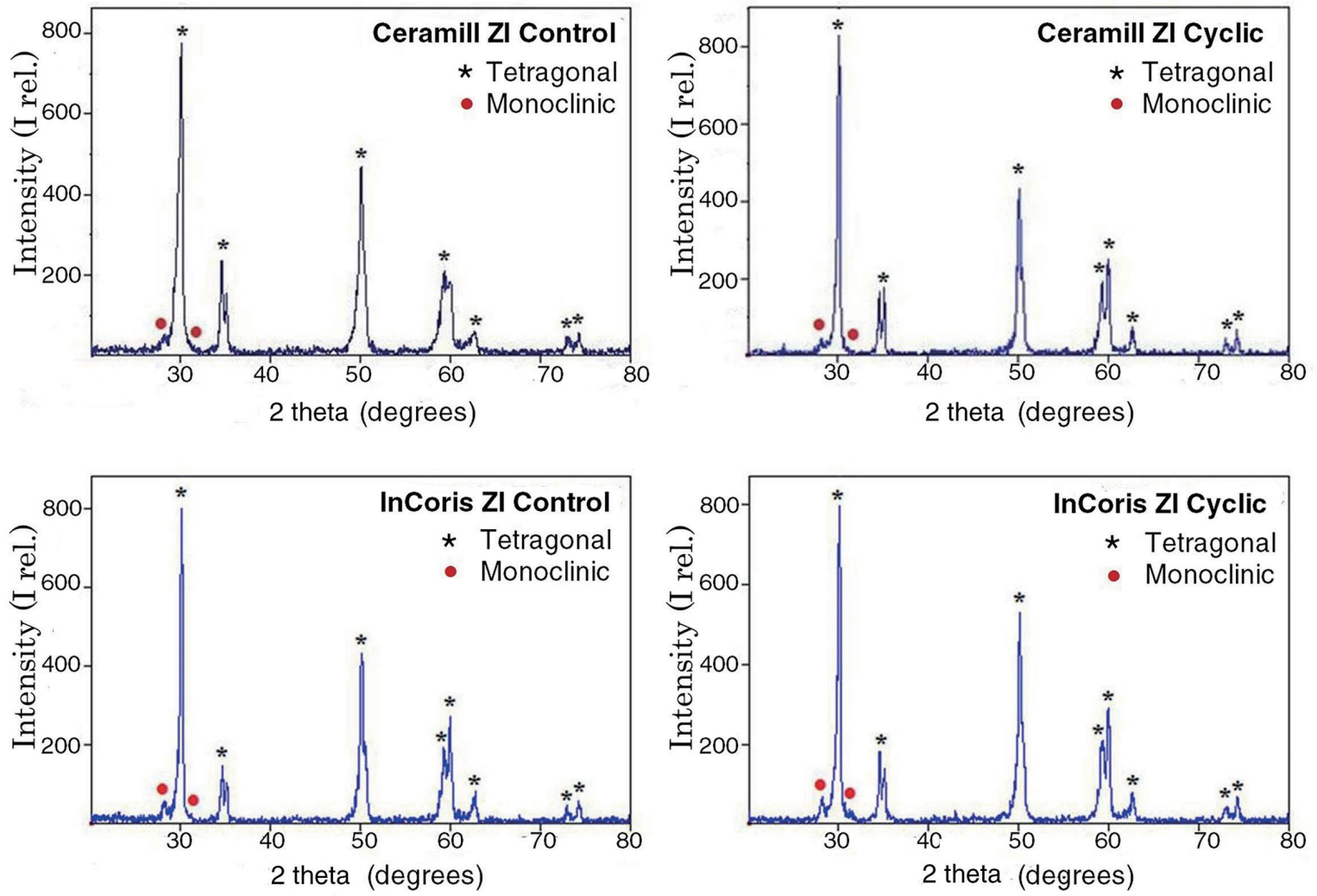

Fig. 3. $\mu X R D$ pattern of representative specimens obtained from the surface of zirconia specimens. 
Table 2. Monoclinic phase content (\%) of control and test abutments prepared from two Y-TZP ceramics and assessed by three different techniques

\begin{tabular}{clccccc}
\hline \multirow{2}{*}{ Materials } & \multicolumn{2}{c}{ XRD (wt\%) } & \multicolumn{2}{c}{ Micro XRD (wt\%) } & \multicolumn{2}{c}{ Micro-Raman (vol\%) } \\
& Control & Cyclic loading & Control & Cyclic loading & Control & Cyclic loading \\
\hline Ceramill ZI & $2.79(0.8)^{\mathrm{A}}$ & $4.31(2.0)^{\mathrm{a}}$ & $2.83(1.11)^{\mathrm{B}}$ & $4.09(1.42)^{\mathrm{b}}$ & $5.88(4.5)^{\mathrm{C}}$ & $9.1(7.08)^{\mathrm{C}}$ \\
Incoris ZI & $6.66(1.01)^{\mathrm{D}}$ & $7.36(1.07)^{\mathrm{d}}$ & $6.71(1.32)^{\mathrm{E}}$ & $7.59(1.25)^{\mathrm{e}}$ & $9.96(4.91)^{\mathrm{C}}$ & $11.85(7.38)^{\mathrm{C}}$ \\
\hline
\end{tabular}

Values with different superscripts represent statistically different within each material (cyclic loading effect) or within each column (material type effect).
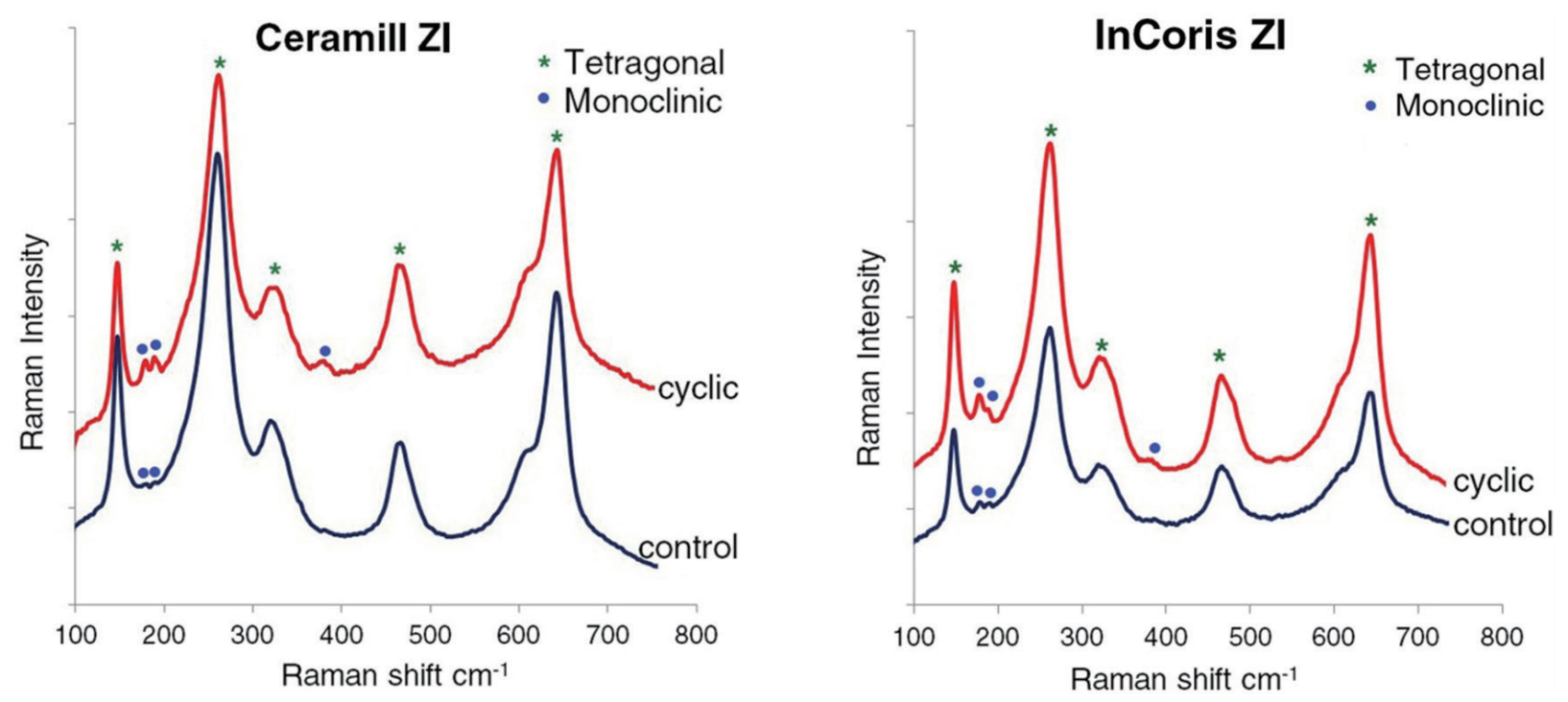

Fig. 4. The representative micro-Raman spectra obtained from the surface of zirconia specimens.

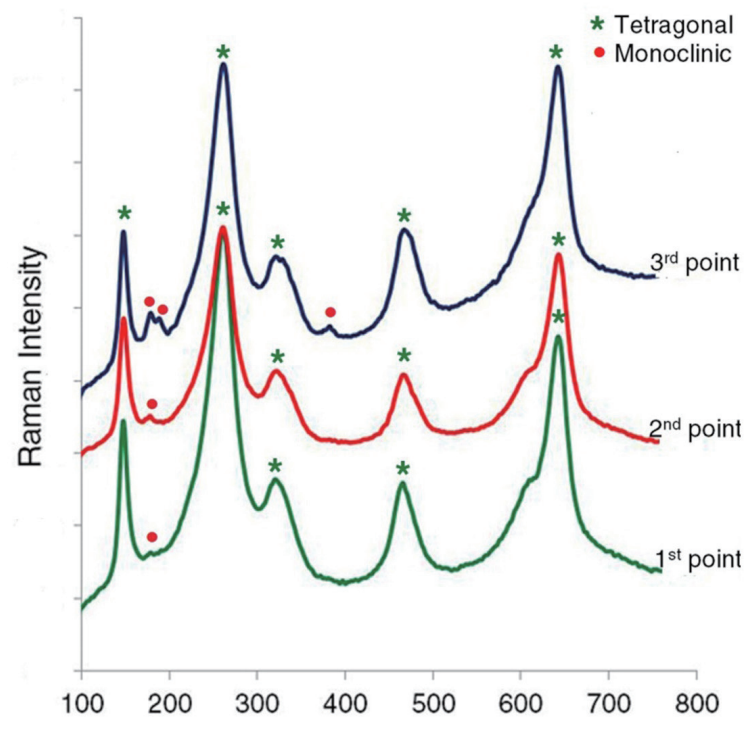

Fig. 5. Micro-Raman spectra obtained from three sequential spots of a cyclically loaded surface. and Ceramill ZI. There was no significant increase in the monoclinic phase after cyclic loading for both InCoris ZI and Ceramill ZI assessed from the micro-Raman technique. The correlations among three quantitative phase analysis methods were also determined using a linear regression model. A positive agreement was achieved between XRD and $\mu$ XRD results $\left(r^{2}=0.81\right)$. However, a large scattered data with very low correlation $\left(\mathrm{r}^{2}=0.1\right)$ was observed between XRD and micro-Raman spectroscopy results.

The surface morphology of the control and cyclically loaded prostheses are shown in Fig. 7. The zirconia prostheses were prepared from two dental milling machines and some differences could be observed from the milled surfaces. The surface of Ceramill ZI control prosthesis was relatively smooth compared with that observed from InCoris ZI control abutment. When observed at higher magnification, some protruded grains were observed on the surface of Ceramill ZI control prosthesis but deep grooves were generally observed on the surface of InCoris ZI control 

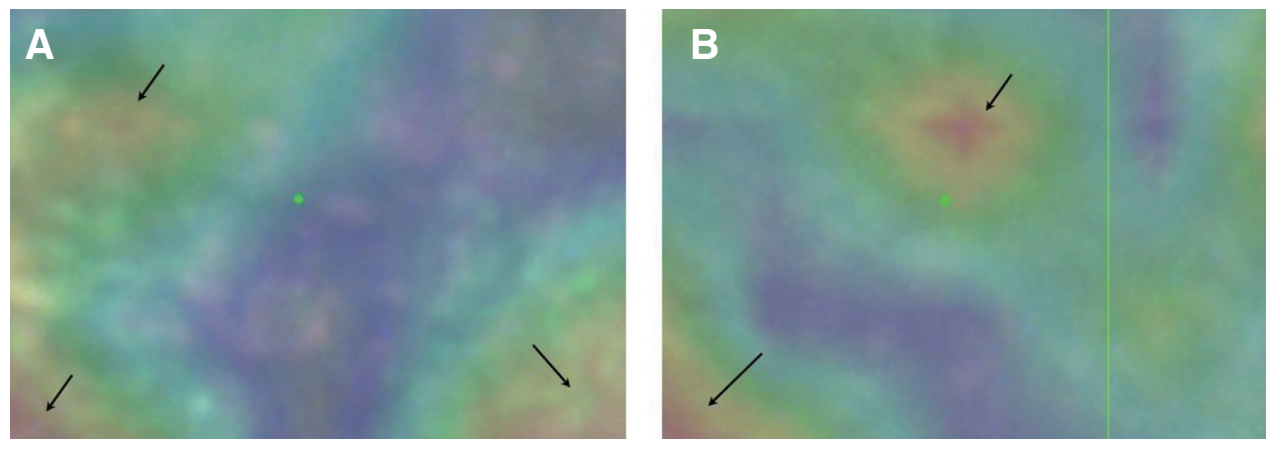

Fig. 6. The Raman spectral images obtained from the cyclic loading area in (A) InCoris ZI (B) Ceramill ZI. The arrows point to the area with strong monoclinic peak intensity.
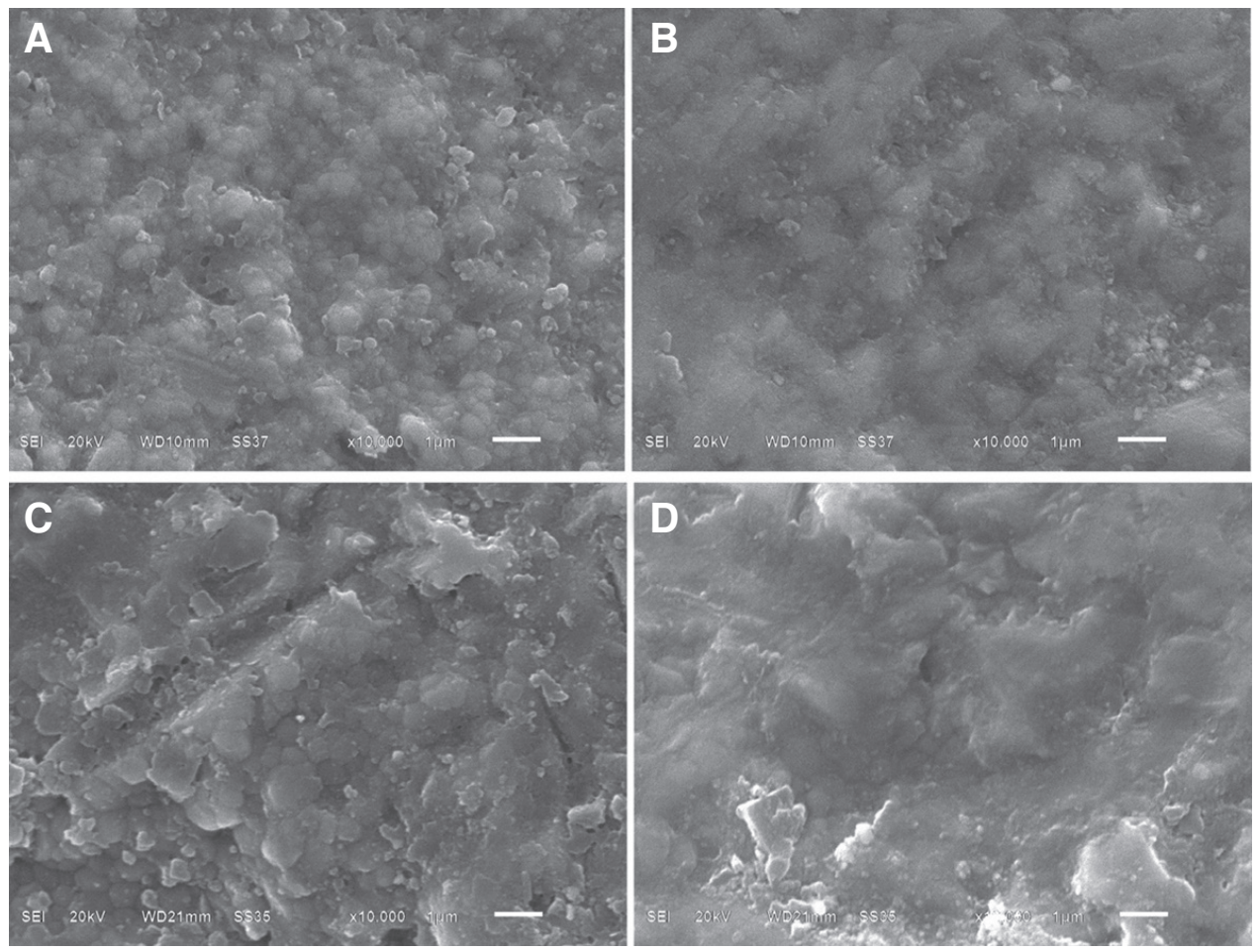

Fig. 7. The representative scanning electron micrographs of Y-TZP abutments: Ceramill Zl control and aged (A and B), InCoris Zl control and aged (C and D).

specimen. The submicron zirconia grains with equiaxial shape were detected in both materials. After cyclic loading, the flattening of the zirconia surfaces was noticeable in both Ceramill ZI and InCoris ZI abutment (Fig. 7B, 7D). Some of the zirconia grains were not clearly distinguished in these areas. No significant crack or fracture line was discerned in the cyclic-loading area. There were also some traces of tungsten carbide material left on the cyclic-loading area.

\section{DISCUSSION}

In this study, the phase transformation of zirconia implantsupported prostheses was assessed after cyclic loading in the presence of moisture at body temperature, to mimic oral environment. Our findings were in agreement with recent studies ${ }^{19-21}$ that cyclic loading could cause an increase in the monoclinic phase content (Table 2). Cotes et al. ${ }^{19}$ observed an increasing trend of monoclinic phase from $0 \%$ to $2.5 \%$ 
after application of $200 \mathrm{~N}$ cyclic loading for 15,000,000 cycles to disc-shaped specimens. The same trend was detected in the study by Egilmez et al., ${ }^{21}$ in which monoclinic phase increased from $13.35 \%$ in control specimens to $16.5 \%$ after cyclic loading for $200 \mathrm{~N}$ for 20,000 cycles. Similarly, Basilio et al..$^{20}$ reported the presence of monoclinic phase contents in four out of eight Y-TZP implant abutment specimens ranging from $14.4 \%$ to $19.3 \%$ after cyclic loading from 11 to $211 \mathrm{~N}$ for 1,000,000 cycles. In this study, the implant-supported prostheses have been made from two well-known CAD-CAM systems. The testing conditions were set-up to simulate function in the oral environment. Even these zirconia-based dental ceramics were not affected much in terms of the monoclinic phase transformation after a mechanical loading, but the difference in the material characteristics such as material composition, surface characteristics after milling, and topographical surface alteration after cyclic loading would provide some useful information for dental clinical use on how these materials would perform during function.

Comparing between materials, InCoris ZI exhibited greater amounts of monoclinic phase than Ceramill ZI for both control and cyclically loaded groups. The difference in monoclinic fraction of these two materials could be due to the different material formulation and processing procedures carried out by two different CAD/CAM systems. Technically the monoclinic phase transformation depends on the grain size and shape, ${ }^{22,23}$ which in turn is influenced by the composition and processing conditions. ${ }^{24}$ Therefore, different composition and sintering procedures recommended by different manufacturers could cause the differences in the microstructures and monoclinic contents before and after cyclic loading of the two materials used in this study.

For the ageing assessment techniques, the monoclinic phase content obtained from the micro-Raman technique appeared to be higher than those obtained from XRD and $\mu \mathrm{XRD}$ techniques (Table 2). This difference might be due to the differences in the nature of each assessment technique. $\mathrm{XRD}$ is a traditional method used for quantitative assessment of the phase transformation in zirconia ceramics. Several studies have used XRD method to assess the phase transformation caused from zirconia ageing procedures. ${ }^{10-14}$ It is a nondestructive technique and should be considered as the initial step for assessment of ageing sensitivity of zirconia samples. However, it would be difficult to obtain accurate information when the small amount of transformation occurs, as the accuracy of measurement is restricted by the signal-to-noise ratio especially where the monoclinic fraction is less than $5 \% .{ }^{25}$ In addition, a local information is not available with a conventional XRD, which generally characterizes the overall components of a sample with an $\mathrm{x}$-ray beam scanning over the whole surface of a specimen, while the results can vary at different locations on the same specimen surface. ${ }^{25}$ Therefore, the results obtained from the $\mathrm{XRD}$ analysis would provide an average value of all phases. The cyclic loading in this study also would not produce homogenous phase transformation throughout the speci- men surface and the signal-to-noise ratio was optimized by increasing the step time.

The $\mu \mathrm{XRD}$ used in this study utilizes a parallel beam optics with exchangeable pin hole collimator that can focus directly to the cyclic-loading area within the diameter of 100 $\mu \mathrm{m}$. The parallel beam technique provides a higher intensity beam and improved resolution than that obtained from a divergent beam configuration. ${ }^{26}$ However, one of the drawbacks of small spot sizes is prolonged measurement time and possible poor crystallite statistics in the evaluated volume, dependent on the size of the crystallites. Use of two dimensional detector systems could solve this problem because the most noticeable advantage of GADDS is saving the data collection time with better diffraction profiles when compared with the diffraction profiles collected by a conventional diffractometer. ${ }^{27,28}$

In this study, the $\mu \mathrm{XRD}$ was used to examine the exact area of a stressed surface. A satisfactory agreement was observed between XRD and $\mu$ XRD. While the amount of the transformed monoclinic phase was very minor, the area of the transformed zone appeared to be in a micrometer level as shown in Fig. 6. As the focusing diameter of the $\mu \mathrm{XRD}$ beam was in a $100 \mu \mathrm{m}$ range, the results appeared to be comparable either examining the whole surface or a 100 $\mu \mathrm{m}$-diameter area because the phase to be detected was very small in size and very little in quantity. Nevertheless, the $\mu X R D$ would be a better tool for assessment of localized small area in a large specimen especially when we would like to assess the effect of cyclic loading from a relatively large occlusal surface of dental crowns.

Raman spectroscopy can be used as an alternative to XRD for phase transformation quantification. ${ }^{29,30}$ Both the tetragonal and monoclinic variants of zirconia have very strong Raman signals with distinct and characteristic vibrational spectra. The advantage of Raman spectroscopy over the XRD is its better spatial resolution, which provides information about not only transformation at the surface but diffusion into the bulk of the material. ${ }^{29}$ Moreover, as monoclinic zirconia shows a stronger Raman scattering than tetragonal one, the Raman spectroscopy can be a useful tool in detecting the amount of monoclinic phase, especially when the monoclinic content is low. ${ }^{31}$ In this study, the micro-Raman spectra were obtained from the spots on a specimen surface. A cyclic loading was performed at a specific area and a phase transformation occurred locally. The lower stress level and 100,000 cycles of cyclic loading used in this study induced just a small amount of phase transformation. Since the area assessed with this technique was in the order of a micrometer diameter, the resultant peak intensity depended on the point where the spectrum was collected, i.e., one point might catch the monoclinic spot while the other might not be. The representative result obtained from three different points is shown in Fig. 5 and it would be well explained by the Raman spectral images displayed in Fig. 6. However, it should be noted that the spectra collected from the points with strong monoclinic signal was used to represent the tetragonal-to-monoclinic 
transformation. If the transformation was homogeneous in the affected area, the spectrum collected from any point would yield a comparable result. On the other hand, when the transformation was inhomogeneous, there would be points with low or high monoclinic peak intensity as observed in this study. Even so, the Raman spectroscopy appeared to be a suitable analysis tool to identify the effect of a mechanical loading in this study.

Comparing between the assessment techniques, MuñozTabares $e t$ al..$^{32}$ compared the amount of monoclinic phase assessed by XRD and the prediction amount on the surface using data from Raman spectroscopy measured at different depth on the cross section of hydrothermal degraded Y-TZP samples at different ageing times. They reported that the satisfactory agreement was observed for ageing time more than 10 hours where there was large transformation. However, for shorter ageing times where transformation was not homogenous, the results from XRD were always higher than that calculated from the Raman spectroscopy data. Likewise, Siarampi et al. ${ }^{33}$ studied the effect of hydrothermal ageing on the phase transformation and flexural strength of two zirconia ceramics. Monoclinic phase fraction was quantitatively evaluated by XRD and Raman spectroscopy and higher monoclinic values were extracted by $\mathrm{XRD}$ than the latter one. In this study, the monoclinic volume fractions assessed by XRD and $\mu$ XRD were lower than that obtained by the micro-Raman and the correlations between monoclinic content obtained from XRD and micro-Raman were disproved. The reasons for this disparity could be explained by the difference in sample volume that each assessment technique explored for crystalline content calculation. Also for the micro-Raman analysis, the point with strong monoclinic signal was used to represent monoclinic content generated in that area. As a result, the results obtained from the micro-Raman analysis were not the average values, and they would reasonably be higher than those obtained from XRD and $\mu$ XRD techniques in both control and cyclic loading groups.

The surface area assessed by XRD, $\mu \mathrm{XRD}$, and microRaman spectroscopy were different. While the XRD technique scanned the entire surface of a specimen, the focusing area of the $\mu$ XRD beam was only $100 \mu \mathrm{m}$ in diameter. The focusing spot of the micro-Raman analysis was even smaller. The depth of $\mathrm{x}$-ray penetration into a surface from XRD and $\mu \mathrm{XRD}$ is $\sim 13 \mu \mathrm{m}^{34}$ and was considered to be farther than that of the micro-Raman analysis $(<5 \mu \mathrm{m}) .^{35}$ Therefore, the sample volume explored by the micro-Raman technique was the smallest when compared with those assessed with XRD and $\mu$ XRD. In this study, cyclic loading between 20 and 98 $\mathrm{N}$ for 100,000 cycles under distilled water at body temperature $\left(37^{\circ} \mathrm{C}\right)$ produced an increase in monoclinic phase fraction with an inhomogeneous tetragonal-to-monoclinic transformation on the zirconia loading surface. With the small amount of monoclinic content, an assessment of a small or a large surface area to point out the monoclinic spots would make a significant difference as evaluating the whole surface would yield a number of monoclinic as an average value.
While examining the transformation grain would be more difficult and the result might be more specific, the nature of transformation should be identified beforehand in order to correctly interpret the outcome. In this study, investigation of a small cyclically loaded area to point out the monoclinic spot would be more preferable. The advantage of using $\mu X R D$ or micro-Raman spectroscopy over the use of XRD could not be clearly identified in this study, but it was more reasonable to use $\mu \mathrm{XRD}$ or micro-Raman spectroscopy to investigate the area of interest, not the entire specimen surface.

In this laboratory study, we performed cyclic loading on the complex geometric anatomical samples in the presence of moisture at body temperature to make it clinical resemblance. However, there are some limitations in our study. According to the study of normal chewing force by FontijnTekamp et al., ${ }^{36}$ the load of $98 \mathrm{~N}$ in our study corresponds to the physiological loading of maxillary anterior teeth only. Besides, the number of stress cycles we used here represent just the initial clinical service periods. To understand the ageing behavior of dental zirconia that covers over all dental prosthesis, extended upper stress limit and more number of mechanical cycling are necessary. Apart from this, dental restorations are also susceptible to the effects of thermal changes and $\mathrm{pH}$ variations in the oral environment, and ageing behavior of zirconia in response to these factors should also be studied.

\section{CONCLUSION}

Based on the findings of our study, the following conclusions can be drawn. Mechanical cyclic loading in the presence of moisture at body temperature produced a significant phase transformation in Y-TZP prostheses fabricated from two different CAD/CAM systems. For the quantitative assessment of a phase transformation of zirconia-based dental ceramics, the results obtained from XRD and $\mu$ XRD were comparable. However, $\mu \mathrm{XRD}$ or micro-Raman spectroscopy could be used to investigate the area of interest more appropriately when the studied area was small compared with the specimen surface.

\section{ORCID}

Phyu Phyu Kyaw bttps://orcid.org/0000-0002-3071-2071

Kallaya Suputtamongkol https://orcid.org/0000-0003-2933-0516

\section{REFERENCES}

1. Andersson B, Odman P, Lindvall AM, Lithner B. Single-tooth restorations supported by osseointegrated implants: results and experiences from a prospective study after 2 to 3 years. Int J Oral Maxillofac Implants 1995;10:702-11.

2. Kimura H, Horng CJ, Okazaki M, Takahashi J. Oxidation effects on porcelain-titanium interface reactions and bond strength. Dent Mater J 1990;9:91-9.

3. Gomes AL, Montero J. Zirconia implant abutments: a review. 
Med Oral Patol Oral Cir Bucal 2011;16:e50-5.

4. Denry I, Kelly JR. State of the art of zirconia for dental applications. Dent Mater 2008;24:299-307.

5. Kelly JR, Denry I. Stabilized zirconia as a structural ceramic: an overview. Dent Mater 2008;24:289-98.

6. Chevalier J, Gremillard L, Virkar AV, Clarke DR. The tetragonal-monoclinic transformation in zirconia: Lessons learned and future trends. J Am Ceram Soc 2009;92:1901-20.

7. Chevalier J, Cales B, Drouin JM. Low-temperature aging of Y-TZP ceramics. J American Ceram Soc 1999;82:2150-4.

8. Hummer CD 3rd, Rothman RH, Hozack WJ. Catastrophic failure of modular zirconia-ceramic femoral head components after total hip arthroplasty. J Arthroplasty 1995;10:848-50.

9. \#2 FPSNS. Hip implant recall because of potential fracture problems. http://www.fda.gov/downloads/Safety/FDAPatient SafetyNews/UCM417762.pdf; 2002.

10. Flinn BD, deGroot DA, Mancl LA, Raigrodski AJ. Accelerated aging characteristics of three yttria-stabilized tetragonal zirconia polycrystalline dental materials. J Prosthet Dent 2012;108: 223-30.

11. Kohorst P, Borchers L, Strempel J, Stiesch M, Hassel T, Bach FW, Hübsch C. Low-temperature degradation of different zirconia ceramics for dental applications. Acta Biomater 2012;8:1213-20.

12. Cattani-Lorente M, Scherrer SS, Ammann P, Jobin M, Wiskott HW. Low temperature degradation of a Y-TZP dental ceramic. Acta Biomater 2011;7:858-65.

13. Kim HT, Han JS, Yang JH, Lee JB, Kim SH. The effect of low temperature aging on the mechanical property \& phase stability of Y-TZP ceramics. J Adv Prosthodont 2009;1:113-7.

14. Flinn BD, Raigrodski AJ, Singh A, Mancl LA. Effect of hydrothermal degradation on three types of zirconias for dental application. J Prosthet Dent 2014;112:1377-84.

15. Sailer I, Fehér A, Filser F, Gauckler LJ, Lüthy H, Hämmerle $\mathrm{CH}$. Five-year clinical results of zirconia frameworks for posterior fixed partial dentures. Int J Prosthodont 2007;20:383-8.

16. Zembic A, Philipp AO, Hämmerle CH, Wohlwend A, Sailer I. Eleven-year follow-up of a prospective study of zirconia implant abutments supporting single all-ceramic crowns in anterior and premolar regions. Clin Implant Dent Relat Res 2015;17:e417-26.

17. ISO 14801. Dentistry - Implants - Dynamic fatigue test for endosseous dental implants. 2nd ed. International Standards Organization (ISO); Geneva; Switzerland, 2007. https://www. iso.org/standard/41034.html

18. Munoz-Tabares AMa, Anglada MJ. Quantitative analysis of monoclinic phase in 3Y-TZP by raman spectroscopy. J Am Ceram Soc 2010;93:1790-5.

19. Cotes C, Arata A, Melo RM, Bottino MA, Machado JP, Souza RO. Effects of aging procedures on the topographic surface, structural stability, and mechanical strength of a ZrO2-based dental ceramic. Dent Mater 2014;30:e396-404.

20. Basílio Mde A, Cardoso KV, Antonio SG, Rizkalla AS, Santos Junior GC, Arioli Filho JN. Effects of artificial aging conditions on yttria-stabilized zirconia implant abutments. J Prosthet Dent 2016;116:277-85.

21. Egilmez F, Ergun G, Cekic-Nagas I, Vallittu PK, Lassila LV. Factors affecting the mechanical behavior of Y-TZP. J Mech
Behav Biomed Mater 2014;37:78-87.

22. Lange FF, Dunlop GL, Davis BI. Degradation during aging of transformation-toughened $\mathrm{ZrO} 2-\mathrm{Y} 2 \mathrm{O} 3$ materials at 250 C. J Am Ceram Soc 1986;69:237-40.

23. Schmauder S, Schubert H. Significance of internal stresses for the martensitic transformation in Yttria-stabilized tetragonal zirconia polycrystals during degradation. J Am Ceram Soc 1986;69:534-40.

24. Stawarczyk B, Ozcan M, Hallmann L, Ender A, Mehl A, Hämmerlet $\mathrm{CH}$. The effect of zirconia sintering temperature on flexural strength, grain size, and contrast ratio. Clin Oral Investig 2013;17:269-74.

25. Deville S, Gremillard L, Chevalier J, Fantozzi G. A critical comparison of methods for the determination of the aging sensitivity in biomedical grade yttria-stabilized zirconia. J Biomed Mater Res B Appl Biomater 2005;72:239-45.

26. He BB, Preckwinkel U. X-ray optics for two-dimensional diffraction. Adv in X-ray Anal 2002;45:332-7.

27. He BB. Introduction to two-dimensional X-ray diffraction. Powder Diffraction 2003;18:71-85.

28. He BB. Microdiffraction using two-dimensional detectors. Powder Diffraction 2004;19:110-8.

29. Wulfman C, Sadoun M, Chapelle ML. Interest of raman spectroscopy for the study of dental material: The zirconia material example. IRBM 2010;31:257-62.

30. Clarke DR, Adar F. Measurement of the crystallographically transformed zone produced by fracture in ceramics containing tetragonal zirconia. J Am Ceram Soc 1982;65:284-8.

31. Kim B, Hahn J, Han K. Quantitative phase analysis in tetragonal-rich tetragonal/monoclinic two phase zirconia by Raman spectroscopy. J Mater Sci Lett 1997;16:669-71.

32. Muñoz-Tabares J, Jiménez-Piqué E, Anglada M. Subsurface evaluation of hydrothermal degradation of zirconia. Acta Materialia 2011;59:473-84.

33. Siarampi E, Kontonasaki E, Andrikopoulos KS, Kantiranis N, Voyiatzis GA, Zorba T, Paraskevopoulos KM, Koidis P. Effect of in vitro aging on the flexural strength and probability to fracture of Y-TZP zirconia ceramics for all-ceramic restorations. Dent Mater 2014;30:e306-16.

34. Fillit R, Homerin P, Schafer J, Brugas H, Thevenot F. Quantitative XRD analysis of zirconia-toughened alumina ceramics. J Mater Sci 1987;22:3566-70.

35. Wulfman C, Djaker N, Dupont N, Ruse D, Sadoun M, Chapelle ML. Raman spectroscopy evaluation of subsurface hydrothermal degradation of zirconia. J Am Ceram Soc 2012;95:2347-51.

36. Fontijn-Tekamp FA, Slagter AP, Van Der Bilt A, Van 'T Hof MA, Witter DJ, Kalk W, Jansen JA. Biting and chewing in overdentures, full dentures, and natural dentitions. J Dent Res 2000;79:1519-24. 